\title{
BMJ Open Knowledge, opinions and compliance related to the $100 \%$ smoke-free law in hospitality venues in Kampala, Uganda: cross-sectional results from the KOMPLY Project
}

Shannon Gravely, ${ }^{1}$ Kellen Namusisi Nyamurungi, ${ }^{2}$ Steven Ndugwa Kabwama, ${ }^{3,4}$ Gabriel Okello, ${ }^{5}$ Lindsay Robertson, ${ }^{6}$ Kelvin Khow Chuan Heng, ${ }^{7}$ Achiri Elvis Ndikum, ${ }^{8}$ Adeniyi Samuel Oginni, ${ }^{9}$ Jean Christophe Rusatira, ${ }^{10}$ Socrates Kakoulides, ${ }^{11}$ Mark D Huffman, ${ }^{12}$ Salim Yusuf, ${ }^{13}$ Eduardo Bianco ${ }^{14}$

To cite: Gravely S,

Nyamurungi KN, Kabwama SN, et al. Knowledge, opinions and compliance related to the $100 \%$ smoke-free law in hospitality venues in Kampala, Uganda: cross-sectional results from the KOMPLY Project. BMJ Open 2018;8:e017601. doi:10.1136/ bmjopen-2017-017601

- Prepublication history for this paper is available online. To view these files, please visit the journal online (http://dx.doi. org/10.1136/bmjopen-2017017601).

Received 5 May 2017

Revised 12 September 2017

Accepted 21 September 2017

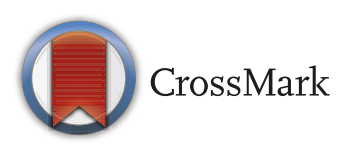

For numbered affiliations see end of article.

Correspondence to

Dr Shannon Gravely;

Shannon.gravely@uwaterloo.ca

\section{ABSTRACT}

Objective This study evaluated knowledge, opinions and compliance related to Uganda's comprehensive smoke-free law among hospitality venues in Kampala Uganda.

Design This multi-method study presents cross-sectional findings of the extent of compliance in the early phase of Uganda's comprehensive smoke-free law (2 months postimplementation; pre-enforcement).

Setting Bars, pubs and restaurants in Kampala Uganda. Procedure and participants A two-stage stratified cluster sampling procedure was used to select hospitality sites stratified by all five divisions in Kampala. A total of 222 establishments were selected for the study. One hospitality representative from each of the visited sites agreed to take part in a face-to-face administered questionnaire. A subsample of hospitality venues were randomly selected for tobacco air quality testing $(n=108)$. Data were collected between June and August 2016.

Outcome measures Knowledge and opinions of the smoke-free law among hospitality venue staff and owners. The level of compliance with the smoke-free law in hospitality venues through: (1) systematic objective observations (eg, active smoking, the presence of designated smoking areas, 'no smoking' signage) and (2) air quality by measuring the levels of tobacco particulate matter $\left(\mathrm{PM}_{2.5}\right)$ in both indoor and outdoor venues.

Results Active smoking was observed in $18 \%$ of venues, $31 \%$ had visible 'no smoking' signage and $47 \%$ had visible cigarette remains. Among interviewed respondents, $57 \%$ agreed that they had not been adequately informed about the smoke-free law; however, $90 \%$ were supportive of the ban. Nearly all respondents $(97 \%)$ agreed that the law will protect workers' health, but $32 \%$ believed that the law would cause financial losses at their establishment. Indoor $\mathrm{PM}_{2.5}$ levels were hazardous $\left(267.6 \mu \mathrm{g} / \mathrm{m}^{3}\right)$ in venues that allowed smoking and moderate $\left(29.6 \mu \mathrm{g} / \mathrm{m}^{3}\right)$ in smoke-free establishments.

\section{Strengths and limitations of this study}

- This multi-method study included a systematic measure (observational checklist), an objective measure (tobacco air quality, particulate matter $\left(\mathrm{PM}_{2.5}\right)$ ) as well as subjective data (face-toface administered questionnaire) among a large sample of hospitality venues in Uganda's capital city, Kampala.

- $\mathrm{PM}_{2.5}$ is useful for determining the concentration of tobacco smoke in the environment because it is a well-established, validated marker for secondhand smoke, has been used extensively in hospitality venues and it provides real-time, low-cost data using a portable, quiet instrument.

- However, although $\mathrm{PM}_{25}$ is commonly used, it is not as sensitive as measuring air nicotine, which is a specific indicator of tobacco smoke pollution. Tobacco pollution levels in the environment may be compromised by other environmental conditions. However, air nicotine and $\mathrm{PM}_{2.5}$ measurements are highly correlated and air nicotine measurements are expensive.

- The findings from the survey interviews could have been subject to social desirability and not reflect true opinions or compliance practices among all respondents. All participants were however assured that their responses were anonymous in order to reduce this bias.

- The findings of this study cannot be generalised to other geographical locations in Uganda, such as other cities or rural areas outside of Kampala.

Conclusions In the early phase of Uganda's smoke-free law, the level of compliance in hospitality venues settings in Kampala was suboptimal. Civil society and the media have strong potential to inform and educate the hospitality industry and smokers of the benefits and requirements of the smoke-free law. 


\section{BACKGROUND}

Secondhand smoke (SHS) is recognised throughout the world as a significant cause of premature death and disease as it contains more than 7000 chemical compounds, 250 of which are harmful and over 60 are carcinogenic. ${ }^{1}$ There is strong scientific evidence that links SHS to the development of non-communicable diseases, particularly heart disease, lung cancer and other respiratory illnesses as well as health problems in infants and children. ${ }^{2}$ Notably, it is estimated that approximately 890000 non-smokers die each year from SHS exposure. ${ }^{3}$ Therefore, a key intervention in reducing the burden of tobacco smoking-attributable morbidity and mortality is to protect all people from exposure to SHS.

Comprehensive smoke-free laws are among the most effective tobacco control strategies available. ${ }^{4}$ The strong implementation of smoke-free laws has been shown to significantly reduce or eliminate SHS in key public venues (thus drastically improving air quality), ${ }^{5}$ help smokers quit and reduce tobacco-related illnesses, hospitalisations, deaths and healthcare costs. ${ }^{4-8}$ Moreover, empirical studies using objective economic indicators have found that smoke-free policies do not have negative economic consequences for businesses, including restaurants and bars, with a positive effect observed in some studies. ${ }^{9}$ Particularly, smoke-free policies are associated with increased worker productivity and reduced overhead costs (eg, cleaning and insurance) ${ }^{9}$ Notably, of all public places, restaurants, bars, pubs and nightclubs have the highest SHS concentrations. ${ }^{10-12}$ Moreover, hospitality workers absorb considerable amount of SHS and often experience greater respiratory symptoms compared with workers in other public settings. ${ }^{13}$

There are a plethora of studies, spanning multiple countries across all continents (eg, Americas, Asia and Europe and now more recently Africa) which have shown that strong smoke-free laws lead to dramatic reductions in indoor air pollution in public places ${ }^{14-18}$ and that locations that do not have smoking regulations have very high levels of SHS ${ }^{18}{ }^{19}$ For example, in a 2008 study of 32 countries, the level of tobacco-related fine particulate matter of diameter less than $2.5 \mu \mathrm{m}\left(\mathrm{PM}_{2.5}\right)$-a validated atmospheric marker for the presence of SHS ${ }^{1620}$-was on average $87 \%$ lower in countries with comprehensive smoke-free laws in comparison to countries without such laws. ${ }^{21}$ Additionally, a recent study carried out in Ghana (where there is no national smoke-free law and smoking is allowed in designated smoking areas in indoor public places and workplaces), ${ }^{19}$ showed that smoking venues had markedly elevated $\mathrm{PM}_{2.5}$ compared non-smoking venues (median $553 \mu \mathrm{g} / \mathrm{m}^{3}$ vs $16 \mu \mathrm{g} / \mathrm{m}^{3}$, respectively) and also higher hair nicotine concentrations in non-smoking employees working in venues that allowed smoking compared with non-smoking establishments (median $2.49 \mathrm{ng} / \mathrm{mg}$ vs median $0.16 \mathrm{ng} / \mathrm{mg}$, respectively).

Although between $30 \%$ and $40 \%$ of high-income and middle-income countries have implemented comprehensive smoke-free policies, many low income countries have not, ${ }^{22}$ specifically in the African region. ${ }^{23}$ This is disconcerting, considering about $90 \%$ of people in Africa are unprotected from SHS. ${ }^{23}$ By 2014, only five African countries had implemented a complete smoking ban in all public places, eight countries had banned smoking in restaurants and nine in pubs and bars. ${ }^{23}$ However, even in locations where smoking is prohibited by law, exposure to SHS in some public places is still high as compliance tends to be low (eg, by allowing designated smoking areas). A study in Kenya, Zambia and Mauritius (which comprised a sample of 4251 smokers) reported that active smoking in bars was $83 \%, 70 \%$ and $45 \%$, respectively, despite smoking bans in these venues. ${ }^{24}$

In May 2016, Uganda became one of the only African countries to become $100 \%$ smoke-free in all public places after implementation of the 2015 Tobacco Control (TC) Act..$^{25}$ In brief, the 2015 TC Act and the respective measures apply to all of Uganda; there are no regional variations. This national legislation includes the prohibition of smoking in all public places, workplaces, means of transport and other outdoor spaces within $50 \mathrm{~m}$. The 2015 TC Act also includes that all smoking cues, such as ashtrays, shall be removed from all public indoor locations and outdoor areas where smoking is prohibited (within $50 \mathrm{~m}$ of any window, door or intake mechanism). The person responsible for the premises must display a no-smoking sign that is conspicuous, clear, prominent and representative of all languages spoken in the area (including English and Swahili). The 2015 TC Act additionally outlines liability and fines for any violations (non-compliance).

Although the $100 \%$ smoke-free law had been implemented at the time of data collection for this study (2 months postimplementation), there was no governing body that was enforcing the smoke-free policy, as the Uganda Ministry of Health had not finalised the regulations of the law. Generally, public establishments volunteered to comply with the law until enforcement became fully organised and mandatory (approximately 8 months postimplementation).

In $2013,10.3 \%$ of men and $1.8 \%$ of women $(0.9$ million adults) smoked tobacco in Uganda ${ }^{26}$ and consumption is projected to rise. Interestingly, only $75 \%$ of adult smokers believe that breathing other peoples' smoke causes serious illness in non-smokers. ${ }^{26}$ Therefore, the time is ripe to ensure the implementation of strong tobacco control measures in Uganda, most notably, smoke-free bans in all public places. To the best of our knowledge, there is no published research on compliance with the smoke-free law in Uganda and, specifically, there have been no studies objectively assessing the level of SHS exposure by measuring the levels of $\mathrm{PM}_{2.5}$. As SHS exposure is high in Ugandan hospitality venues (in 2013 an estimated $62 \%$ of adults who visited bars, pubs or nightclubs were exposed to tobacco smoke) ${ }^{26}$ and there are no surveillance systems for routinely measuring exposure to SHS, the KOMPLY Project was assembled to assess the level of compliance with Uganda's 100\% smoke-free law 
in hospitality venues in Uganda's capital city, Kampala. Specifically, we assessed: (1) knowledge of the 2015 TC Act/new smoke-free legislation and opinions about the $100 \%$ smoke-free law among hospitality venue staff and owners and (2) the level of compliance with the smokefree law in hospitality venues.

\section{METHODS}

\section{Design and setting}

This multi-method study is a cross-sectional analysis of an ongoing pre-postimplementation evaluation project in Kampala, Uganda. The analyses in this initial study evaluated the level of compliance by bars, pubs and restaurants with the $100 \%$ smoke-free law which was implemented on 19 May 2016. Data collection ensued between June and August 2016, approximately 2 months after the date of implementation of the law, but prior to regulated enforcement.

Uganda (officially the Republic of Uganda) is a low-income nation, located in Sub-Saharan, East Africa. It has a population of over 41 million people; half of the population is under the age of 15 and one-fifth of the population is aged $15-24 .{ }^{27}$ Kampala is the capital and largest city of in the Central Region of Uganda and in 2016 the population was recorded as 1.5 million people. ${ }^{28}$

\section{Sample}

The sampling technique used to select hospitality venues (bars, pubs and/or restaurants) was cluster random sampling, with a two-stage sampling procedure. ${ }^{29}{ }^{30}$ This type of sampling methodology is commonly used in population studies and offers many advantages (eg, with regard to geography and feasibility); details have been described elsewhere. $^{30}$

Sample size for the larger study was calculated using the formula by Bennett $e t a l^{29}$ which considers a population divided into clusters. We considered a SE of 0.05 and a confidence level of $95 \%$. A design effect of 2 was set since cluster sampling was used. The probability $(p)$ that hospitality venues would not be compliant with the $100 \%$ smoke-free legislation was set at $49 \%(0.49) .{ }^{31}$ The sample size was calculated as a minimum of 198 ; however, we oversampled and arrived at 222 establishments.

Regionally, Kampala is divided into 99 parishes (clusters) within five divisions. ${ }^{32}$ All divisions and clusters were mapped and then all bars and restaurants were mapped within each cluster and neighbourhood. All clusters had an equal chance of being selected during the sampling process; however, if there was an insufficient number of bars and restaurants in a cluster (fewer than 6), it was excluded from selection and another cluster was randomly selected. For each cluster, the location of the highest concentration of hospitality sites was identified. Overall, 35 clusters were randomly selected from a list using the random ballot method and a minimum of 6 hospitality venues were then randomly chosen from each cluster. For the selection of venues for $\mathrm{PM}_{2.5}$ measurements, a random subsample was drawn from a list of hospitality venues developed at the first stage of the data collection. The number of venues selected from each division was proportional to the number of establishments listed in that division and followed the US Environmental Protection Agency guide to ensure representativeness (eg, for spatial scale of representativeness, appropriate site selection and exposure). ${ }^{33}$

\section{Procedure and data collection}

The overarching study examined knowledge, opinions and compliance related to the smoke-free law; this multi-method study was conducted using three different methods. The first two methods included: (1) a systematic observational checklist and (2) a face-to-face administered questionnaire with a venue owner or staff (waiter, bar-serving staff or manager). These methods were derived from a commonly used guide for assessment of smoke-free law compliance ${ }^{34}$ and an existing survey protocol. ${ }^{32}$

Between 29 June 2016 and 7 July 2016, a checklist was used to gather systematic objective observations of: the number of people in each venue (including how many patrons could be seated in the venue/legaloccupancy), the presence of active smoking and other observations such as the presence of no-smoking signage, ashtrays, discarded cigarette remains, designated smoking areas and so on. At each site, the observation period lasted $20-30 \mathrm{~min}$. Venue staff and patrons were unaware of the study at the time of this first measurement; thus, the process was not disruptive to the ongoing daily operations of the venue. This method was also intended to not influence normal smoking behaviour. At the end of each site observation, the research fieldworkers approached an owner or manager and asked for their participation in a face-to-face survey interview to assess their: (1) knowledge of the new smoke-free legislation; (2) opinions related to the $100 \%$ smoke-free law and (3) personal compliance with the smoke-free law. One hospitality representative from each of the 222 visited sites agreed to take part in the interview. The questionnaire on average was approximately $20 \mathrm{~min}$.

The third method of the study included assessments of indoor and outdoor air quality and the presence of SHS that followed an adapted version of the Roswell Park Indoor Air Monitoring Protocol ${ }^{35}$; the US Environmental Protection Agency Speciation Guidance report was used for further guidance. ${ }^{33} \mathrm{PM}_{2.5}$ was used as the marker for SHS concentrations. $\mathrm{PM}_{2.5}{ }_{2.5}$ has commonly been used to measure tobacco PM in various indoor and outdoor public locations across several countries. ${ }^{15-19}{ }^{36-38} \mathrm{PM}_{2.5}$ is a demonstrated atmospheric marker for the presence of $\mathrm{SHS}^{16}$ and the association between airborne nicotine and $\mathrm{PM}_{2.5}$ is high (Spearman's rank correlation=0.71) ${ }^{20}$ Although PM can come from many sources (eg, use of biomass fuel for cooking, heating and outdoor air pollution), tobacco smoking is the most significant source inside buildings where smoking is occurring, particularly in urban settings where electricity is the main source of power. The use of air quality monitoring equipment to 
measure PM concentrations deriving from SHS has been previously validated. ${ }^{39}$

In brief, a subsample of the 222 hospitality establishments $(\mathrm{n}=108)$ was randomly selected and measurements of tobacco-related $\mathrm{PM}_{25}$ distributions were taken with the TSI SidePak Personal Aerosol Monitor AM510. ${ }^{40}$ Fieldworkers visited selected sites at peak time between $6 \mathrm{pm}$ and $12 \mathrm{am}$ and recorded the $\mathrm{PM}_{2.5}$ reading for $90 \mathrm{~min}$. Real-time data on $\mathrm{PM}_{2.5}$ levels were measured over a 4-hour period with $15 \mathrm{~min}$ average exposure, peak exposure and percentage of time when concentrations exceed threshold levels expressed. Air Quality Ratings are derived from the United States Environmental Protection Agency (US EPA) Standard air quality index (AQI) breakpoints (Revised AQI system). ${ }^{5}$

All fieldworkers were trained in the data collection protocol. Informed consent was sought from each of the participants that were interviewed for the study.

\section{Statistical analysis}

Descriptive statistics were performed using cross-tabulation or univariate analyses to obtain frequencies ( $\mathrm{n}$ and \%) of the characteristics of the establishments, the observational checklist items and the questionnaire responses. Data were analysed using Epi Info V.7 (United States Centers for Disease Control and Prevention, Atlanta, Georgia, USA) and Stata V.12 (StataCorp, College Station, Texas, USA).

$\mathrm{PM}_{2.5}$ data were verified and analysed using MS Excel (Microsoft). Average $\mathrm{PM}_{2.5}$ concentration data were generated for each hospitality venue with the minimum, maximum and per cent time over particular health-based thresholds. The means of indoor and outdoor $\mathrm{PM}_{2.5}$ measurements were calculated for each venue. The first and last minute of logged data were removed, because they were averaged with outdoor and entry way air. The remaining data points were averaged to provide an average $\mathrm{PM}_{2.5}$ concentration. The smoking density (the number of burning cigarettes for 100 cubic metres of space $\left(\mathrm{bcs} / \mathrm{m}^{3}\right)$ for each venue was calculated by using the average number of burning cigarettes during the measurement period and the approximate size of the restaurant. The mean and median of each measurement for each type of venue was calculated. Spearman's rank correlation coefficient was used to determine the association of indoor $\mathrm{PM}_{2.5}$ concentrations with smoking density and outdoor $\mathrm{PM}_{2.5}$ concentrations.

\section{RESULTS}

\section{Characteristics of participating hospitality venues}

Overall, 222 hospitality venues were included in this larger study and 108 in the $\mathrm{PM}_{2.5}$ tobacco air quality substudy. The number of sites per division in the larger study ranged from 41 (18.5\%, Central) to $47(21.2 \%$, Makindye) and from 17 (15.7\%, Central and Lubaga) to 29 (26.9\%, Makindye). Among the 222 establishments, half were small (seated 1-50 people) in size (49\%), $65 \%$
Table 1 Characteristics of participating hospitality venues

Subsample ( $n=108)$ for

All hospitality venues $(n=222)$ $\mathrm{PM}_{2.5}$ study*

\begin{tabular}{lcl}
\hline Division, $\mathrm{n}(\%)$ & $41(18.5)$ & $17(15.7)$ \\
Central & $50(22.5)$ & $17(15.7)$ \\
Lubaga & $42(18.9)$ & $20(18.5)$ \\
Kawempe & $42(18.9)$ & $21(19.4)$ \\
Nakawa & $47(21.2)$ & $29(26.9)$ \\
Makindye & & \\
Venue size†, $\mathrm{n}(\%)$ & $108(48.6)$ & $\mathrm{NA}$ \\
Small & $58(26.1)$ & $\mathrm{NA}$ \\
Medium & $56(25.2)$ & $\mathrm{NA}$ \\
Large & & \\
Type of venue, $\mathrm{n}(\%)$ & $133(59.9)$ & $65(60.2)$ \\
Bar/pub & $6(2.7)$ & $1(0.9)$ \\
Restaurant & $81(36.5)$ & $38(35.2)$ \\
Restaurant and bar & $2(0.9)$ & $0(0)$ \\
Other &
\end{tabular}

Nature of venue, $\mathrm{n}(\%)$

\begin{tabular}{|c|c|c|}
\hline Indoor facility (enclosed) & $66(29.7)$ & $33(30.5)$ \\
\hline $\begin{array}{l}\text { Both indoor and outdoor } \\
\text { facility }\end{array}$ & $145(65.3)$ & $68(63.0)$ \\
\hline $\begin{array}{l}\text { Open; non-enclosed/ } \\
\text { partially enclosed facility }\end{array}$ & $11(4.9)$ & $3(2.8)$ \\
\hline \multicolumn{3}{|l|}{ Structure of venue, $\mathrm{n}(\%)$} \\
\hline Permanent structure & $201(90.5)$ & $90(83.3)$ \\
\hline Semipermanent & $16(7.2)$ & $10(9.3)$ \\
\hline Make-shift structure & $5(2.2)$ & $4(3.7)$ \\
\hline
\end{tabular}

Hospitality interview respondents $(n=222)$

Designation of interview respondent, $n(\%)$

\begin{tabular}{lrl} 
Owner & $37(16.7)$ & $32(29.6)$ \\
Manager & $108(48.6)$ & $46(42.6)$ \\
Bar-server or waiter & $75(33.8)$ & $26(24.1)$ \\
Other & $2(0.90)$ & $0(0)$ \\
\hline
\end{tabular}

*Four venues (3.7\%) have missing sample characteristic data. †Measured by how many people can sit in this establishment: $1-50=$ small, $51-100=$ medium, more than $100=$ large.

$\mathrm{NA}$, data not available; PM, particulate matter.

were (open) non-enclosed or partially-enclosed establishments and $90 \%$ were permanent structures (see table 1).

\section{Observational checklist of smoking indicator items}

Figure 1 shows the results of the checklist data. Overall, smoking was observed in $39(18 \%)$ of hospitality sites. One out of every five venues had tobacco products that were visible for sale and $8 \%$ had promotional and/or sponsorship signs and products that were visible. Just over one out of every four establishments (29\%) had signage for a smoking section; however, 79 sites $(36 \%)$ had visible designated smoking areas (with or without signage). Nearly half 


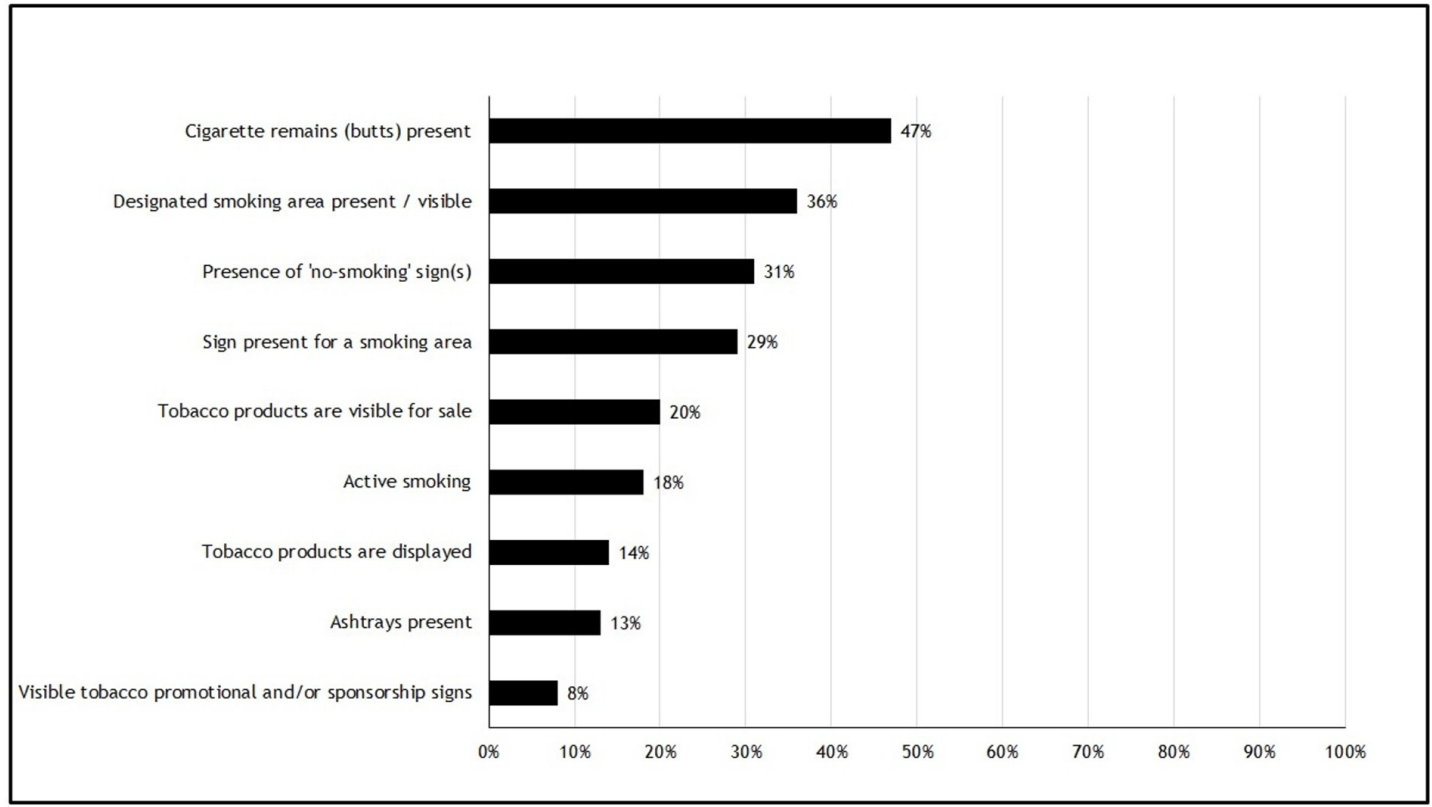

Figure 1 Observational checklist of smoking indicator items among the 222 participating hospitality venues.

of venues had visible smoked cigarette remains (47\%) and $13 \%$ had ashtrays. Notably, while the 100\% smoke-free law in Uganda has preliminary requirements for the size, format, content and placement of 'no smoking' notices, ${ }^{25}$ none of the establishments met these requirements.

\section{Employers' and employees' knowledge and opinions of the $100 \%$ smoke-free law \\ Knowledge}

Figure 2 provides a pictorial summary of the participant's general knowledge about the smoke free law. Among the 222 participants, nearly half (49\%) were venue managers, followed by waiters/barserving staff $(34 \%)$ and owners $(17 \%)$.

Overall, 168 participants (76\%) had heard of Uganda's 2015 TC Act/legislation. Among those who knew of the legislation, awareness of the policy's content was generally low. Awareness that the smoking ban covers all public places was $60 \%(101 / 168)$ and $20 \%(33 / 168)$ knew that the smoke-free law requires the display of regulated no-smoking signage.

Nearly $29 \%$ of the entire sample $(64 / 222)$ either 'strongly agreed' or 'agreed' that they had been adequately informed about the smoke-free law, $14 \%$ were undecided and $57 \%$ disagreed or strongly disagreed (table 2 and figure 2). Less than half knew that there are penalties for smoking violations. Among the 64 respondents that 'agreed' that they has been adequately informed about the smoke-free ban, the majority $(54 / 64=84 \%)$ reported that the media (including social media, television and radio) provided the most information to them. Other responses $(n=10)$ included: newspapers, the government (eg, Ministry of Health), school, patrons (public) and boss (management).

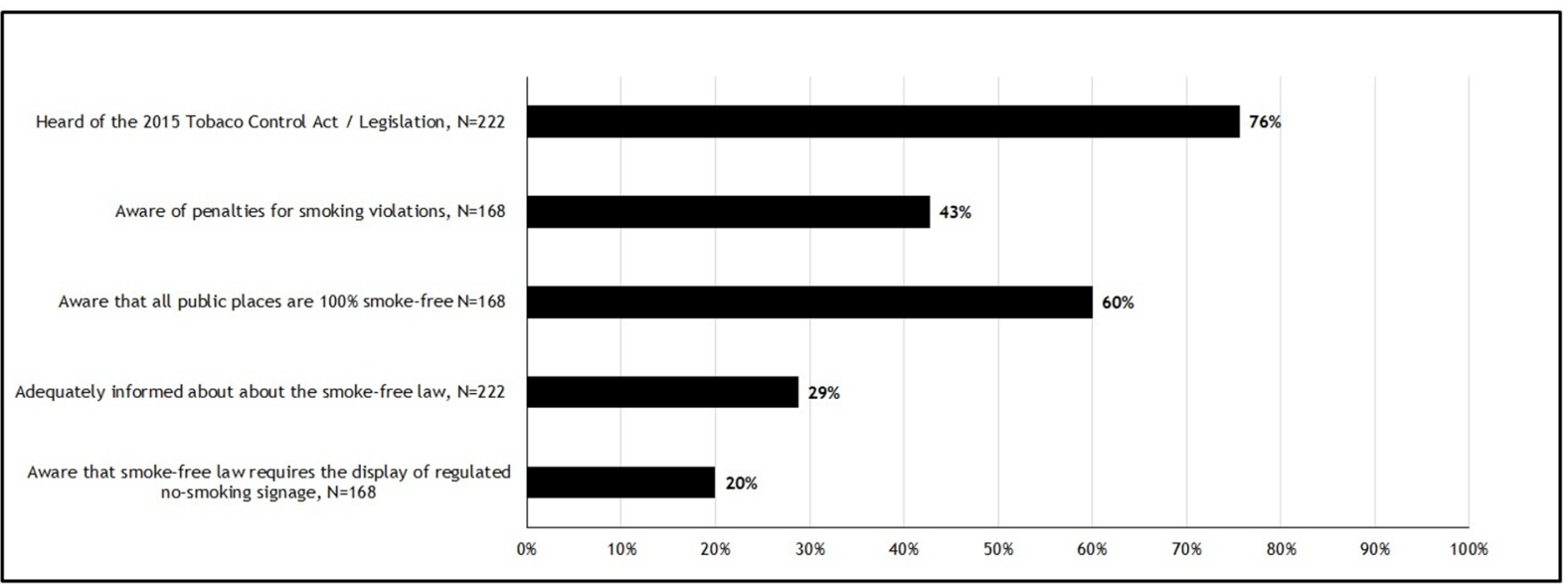

Figure 2 Employers' and employees' knowledge of the Uganda Tobacco Control Act (2015) and the 100\% smoke-free law. N is the number of respondents who were asked the question (denominator). 


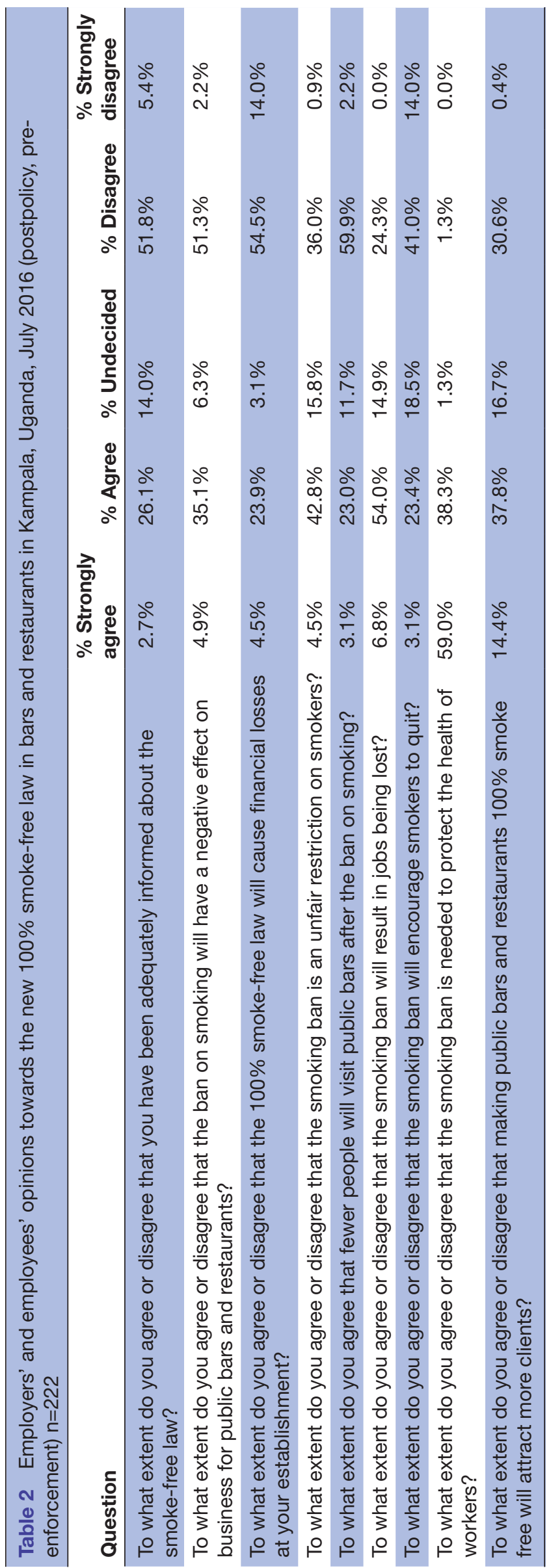

Opinions

Table 2 and figure 3 show the results of the survey of the employers' and employees' opinions about the 100\% smoke-free law.

Nearly all respondents either strongly supported (46\%) or supported $(44 \%)$ the new smoke-free ban in all public places. While $61 \%$ of participants (at least) agreed that the smoke-free law will result in jobs being lost and $40 \%$ 'strongly agreed' or 'agreed' that the law will have a negative effect on hospitality business in general, only $28 \%$ 'strongly agreed' or 'agreed' that the law would cause financial loses at their own establishment. Only about a quarter of participants (27\%) 'strongly agreed' or 'agreed' that the smoking ban in public places will encourage smokers to quit and nearly half $(47 \%)$ 'strongly agreed' or 'agreed' that the smoking ban is an unfair restriction on smokers. Nearly all participants (97\%) 'strongly agreed' or 'agreed' that the law would protect the health of hospitality workers.

When asked 'what are some challenges do you foresee in ensuring that this establishment is $100 \%$ smoke free?', $39 \%$ reported 'minimal assistance from enforcement authorities', 28\% said 'loss of cliental (smokers)', 21\% felt that they would suffer from loss of revenue (eg, partitioning, signage, paying fines, stock) and $10 \%$ said that they would not have the funds for no-smoking signage. More than a quarter of respondents $(29 \%)$ said that they do not foresee any challenges in enforcing the smoke-free law.

\section{Self-reported compliance with Uganda's $100 \%$ smoke-free law}

Table 3 shows the participants' responses to compliance at their hospitality venue.

In brief, $78 \%$ reported that smoking is not allowed in any indoor areas $(19 \%$ reported that smoking is allowed in at least some indoor areas of the venue). When asked 'what do you do in case someone smokes in a place they are not supposed to smoke', $34 \%$ said that they would ask the person to go to the designated smoking area, $38 \%$ would ask the person to stop smoking, $32 \%$ would ask the person to leave the establishment and 13\% would not do anything.

Regarding penalties for violations, $43 \%$ of respondents were aware that there will be penalties for violations if someone was found smoking indoors or on the premises. Finally, when asked if no-smoking signage should be displayed on their premises, $68 \%$ of respondents agreed that signs should be available, but that their establishment did not yet have these signs as required by law.

\section{Average $\mathrm{PM}_{2.5}$ levels in indoor bars and restaurant venues}

Overall, $\mathrm{PM}_{2.5}$ concentrations in 108 venues were collected (figure 4). Among the subsample of 108 hospitality venue visits, venues with indoor and outdoor SHS $(n=84$, $62 \%)$ had average $\mathrm{PM}_{2.5}$ concentration of $171 \mu \mathrm{g} / \mathrm{m}^{3}$ (95\% CI 141.6 to $200.4 \mu \mathrm{g} / \mathrm{m}^{3}$ ) compared with average concentrations of $29.6 \mu \mathrm{g} / \mathrm{m}^{3}$ (95\% CI 25.9 to $32.7 \mu \mathrm{g} /$ $\left.\mathrm{m}^{3}\right)$ for establishments without SHS $(\mathrm{n}=24,22 \%)$. Among enclosed venues that had SHS $(n=67, n=62 \%)$, the average 


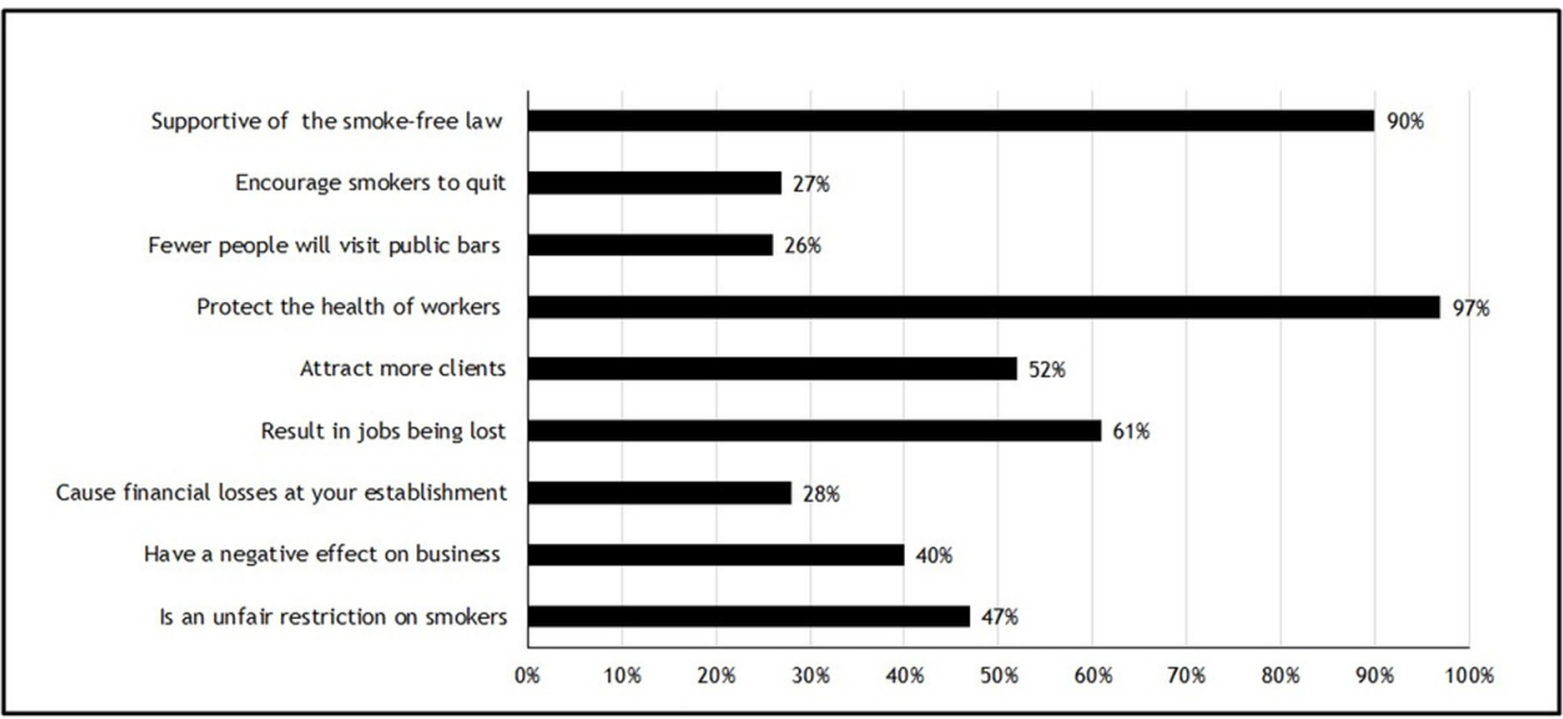

Figure 3 Employers' and employees' opinions about the 100\% smoke-free law.

$\mathrm{PM}_{2.5}$ concentration was $267.6 \mu \mathrm{g} / \mathrm{m}^{3}(95 \%$ CI 233.3 to $312.0 \mu \mathrm{g} / \mathrm{m}^{3}$ ) compared with an average concentration of $85.6 \mu \mathrm{g} / \mathrm{m}^{3}\left(95 \%\right.$ CI 41.1 to $\left.130.1 \mu \mathrm{g} / \mathrm{m}^{3}\right)$ for venues which had open structures and SHS present $(n=17,16 \%)$.

\section{DISCUSSION}

Our findings suggest that in the early phase of Uganda's comprehensive smoke-free law, without enforcement and in the absence of well-defined regulations, compliance in Kampala hospitality venues has been suboptimal. Smoking was observed in one out of every five hospitality sites, 36\% still had a designated smoking area and only a third had 'no smoking' signs or posters. The level of compliance as measured by these indicators was reflected in the air quality readings, such that levels were hazardous in indoor venues that allowed smoking and moderate at smoke-free sites. While more than half of the interviewed respondents self-reported that they were not adequately informed about the law, nearly all $(90 \%)$ were in support of Uganda's new comprehensive smoke-free law.

There is no risk-free level of SHS and even brief exposure can cause immediate harm. ${ }^{26}$ Authoritative sources have consistently recommended and advocated complete comprehensive smoke-free laws with no exceptions (ie, ventilation and smoking areas, whether separately ventilated from non-smoking areas or not, do not reduce exposure to a safe level of risk) to protect workers and the public from SHS exposure. ${ }^{4} 4142$ The findings herein are therefore disconcerting since compliance with the public smoke-free law are not meeting the standards to the WHO Framework Convention on Tobacco Control Article $8^{43}$ (to which Uganda is a Party to the treaty); thus, both smokers and non-smokers remain unprotected against SHS in many Kampala hospitality sites. This state of non-compliance among hospitality venues has also been commonly found in other countries. ${ }^{44-46}$ As there is a significant association between stringency of laws and levels of tobacco smoke pollution, ${ }^{47}$ a strong and coordinated enforcement system is essential for compliance.

Another important factor for successful compliance with a comprehensive smoke-free policy is public support. Changes in public support following the implementation of smoke-free laws may serve as an indicator of changes in societal smoking norms ${ }^{48}$ and widespread public support may facilitate compliance with smokefree laws. ${ }^{49-55}$ Our finding that $90 \%$ of the hospitality employers and employees support Uganda's smoke-free law is therefore encouraging. The 2013 GATS Uganda survey found that the proportion of adults who think smoking should not be allowed in various indoor public places was very high; $98 \%$ reported that smoking should not be allowed in restaurants and $91 \%$ supported smokefree bars. ${ }^{26}$ Notably, the support among Ugandan hospitality venue owners and employees is much higher than previously found in other countries early in the implementation phase of smoke-free legislation. ${ }^{545657}$

Our results are consistent with the misperception of the negative impact of a smoke-free law on business; however, these opinions are not justified, because there is strong evidence that smoke-free policies and regulations do not have an adverse economic impact on the hospitality industry. ${ }^{458}$ In contrast, cigarette smoking imposes significant costs on businesses, which can be considerably reduced if policies that prohibit smoking in the workplace are enacted and strongly enforced. ${ }^{4}$

A comprehensive smoke-free policy is a critical intervention for protecting public health; however, simply implementing the law is not enough. If a smoke-free law is not coupled with a strong enforcement programme, with well-defined regulations, then the law will not have the desired benefits of reducing tobacco use and SHS exposure. $^{18} 21$ Therefore, the Ugandan government 
Table 3 Hospitality interviews: Self-reported compliance with Uganda's 100\% smoke-free law (postpolicy, pre-enforcement)

Survey Question $\%$ Yes

Which of the following best describes the indoor smoking policy at your own establishment?

$\begin{array}{lr}\text { Smoking is allowed anywhere/No policy } & 10.8\end{array}$

Smoking is allowed only in some indoor areas 8.1

Smoking is not allowed in any indoor areas 78.4

Declined to answer 2.7

What do you do in case someone smokes in a place they are not supposed to smoke?

Ask the person to go to the designated smoking area 34.0

Ask the person to stop smoking 38.3

Ask the person to leave the premises 32.4

Do nothing 13.5

Declined to answer 5.9

Are you aware that there will be penalties for violations if someone is caught smoking indoors in public places including bars and restaurants?

\begin{tabular}{l|l}
\hline Yes & 42.8 \\
\hline No & 39.2 \\
\hline I don't know & 18.0 \\
\hline Do you think that no-smoking signs should be displayed on these premises? & 20.7 \\
\hline The smoking signs are already available here & 68.0 \\
\hline Yes, they should be available, but we do not have signs & 5.9 \\
\hline No, they are not necessary & 3.6 \\
\hline Don't know/Not sure & 1.8 \\
\hline Declined to answer & 28.8 \\
\hline Whose responsibility should it be to display no-smoking signs at bars and restaurants? & 20.3 \\
\hline The Ministry of Health & 76.6 \\
\hline Kampala Capital City Authority & 1.4 \\
\hline The owner of the premises & 0.5 \\
\hline Tobacco companies & 0.5 \\
\hline Don't know & \\
\hline Declined to answer & \\
\hline
\end{tabular}

should move swiftly to establish a coordinated enforcement system to facilitate compliance with the smokefree law.

The findings in this study should be interpreted with caution, mainly due to measurement and generalisability. First, tobacco PM was measured using $\mathrm{PM}_{2.5}$; no other SHS markers were collected. It has been shown however that $\mathrm{PM}_{2.5}$ is a well-established marker for $\mathrm{SHS}^{16}$ and it is highly correlated with air nicotine ${ }^{20}$ Second, the findings from the questionnaires could have been subject to social desirability and not reflect true opinions or compliance practices among all respondents. All participants were however assured that their responses were anonymous in order to reduce this bias. Third, the findings cannot be generalised to other geographical locations in Uganda, such as other cities or rural areas outside of Kampala.

There are several strengths of this multi-method study, such as the inclusion of a large number of common types of hospitality venues in Uganda's largest city, Kampala and the use of both objective and subjective measures which provided comprehensive in-depth data about the state of awareness and compliance in the early phase of Uganda's $100 \%$ smoke-free law.

\section{CONCLUSION}

Our findings suggest that 2 months after the implementation of Uganda's comprehensive smoke-free policy-but before enforcement and well-defined regulations - the level of compliance in hospitality venues settings in Kampala was suboptimal. The Ugandan Ministry of Health and Civil Society should campaign for increasing awareness of the smoke-free law and establish a coordinated enforcement system to facilitate compliance with the smoke-free law. Additionally, the use of media (including social media, television and 


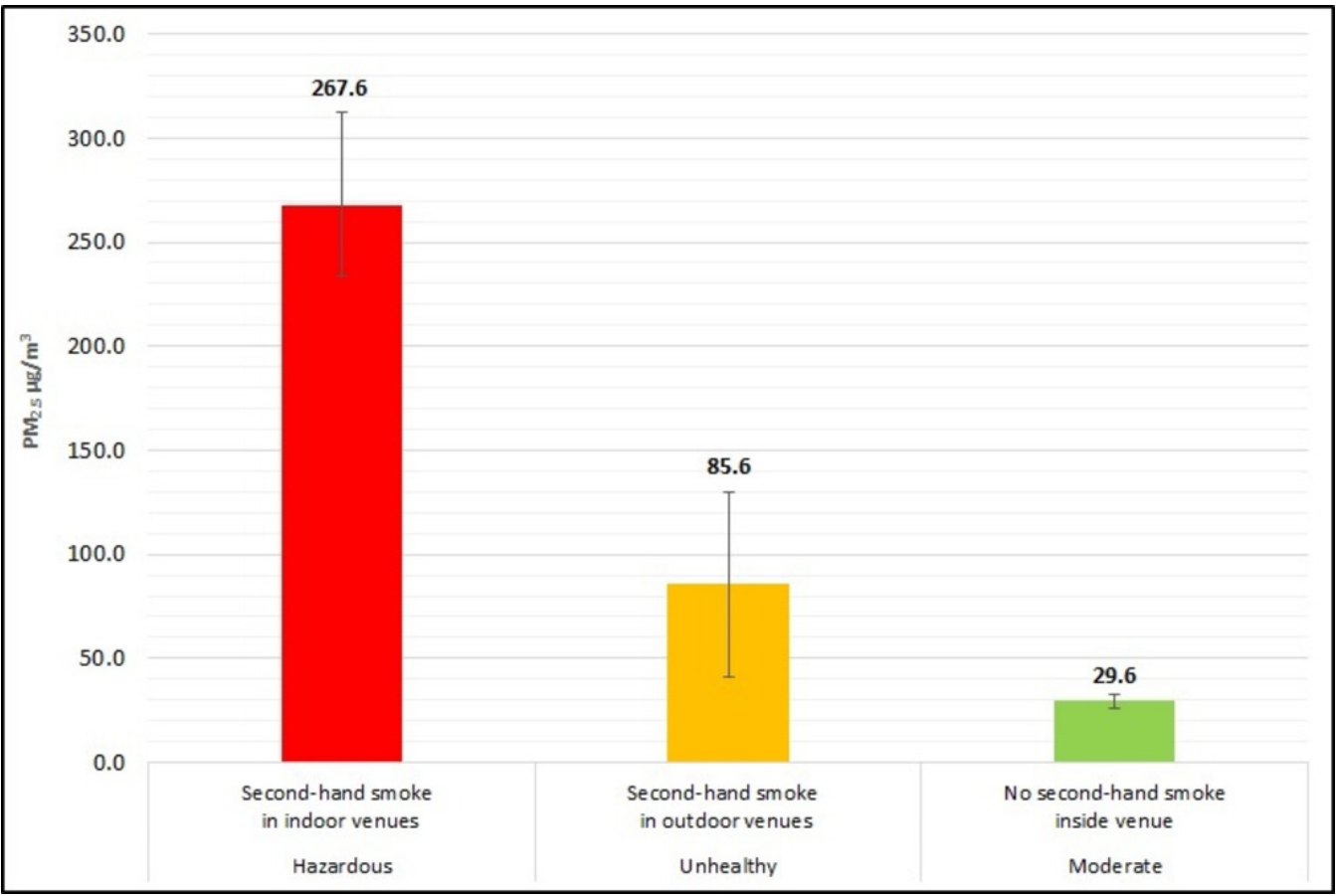

Figure 4 Air quality levels $\mathrm{PM}_{2.5} \mu \mathrm{g} / \mathrm{m}^{3}$ in 108 bars and restaurants in Kampala, Uganda (July-August 2016: postpolicy, preenforcement). Air quality ratings ( $\left(\mathrm{M}_{2.5}\right.$ range) are derived from US EPA Standard AQI breakpoints (Revised AQI system). ${ }^{5} \mathrm{AQI}$, air quality index; PM, particulate matter; USA EPA, United States Environmental Protection Agency.

radio) is a critical avenue to provide information about the $100 \%$ smoke-free law.

\section{Author affiliations}

${ }^{1}$ International Tobacco Control Policy Evaluation (ITC)Project, Department of Psychology, University of Waterloo, Waterloo, Ontario, Canada

${ }^{2}$ Center for Tobacco Control in Africa (CTCA), Kampala, Uganda

${ }^{3}$ School of Public Health, Makerere University, Kampala, Uganda

${ }^{4}$ Mental Health and Substance Abuse, Ministry of Health, Kampala, Uganda

${ }^{5}$ Respiratory Group, Division of Applied Health Sciences, University of Aberdeen,

Aberdeen, UK

${ }^{6}$ Department of Preventive and Social Medicine, University of Otago, Dunedin, New Zealand

${ }^{7}$ World Health Organization, Beijing, China

${ }^{8}$ Association for the Promotion of Youth Leadership, Advocacy and Volunteerism (APYLAV), Yaounde, Cameroon

${ }^{9}$ Nigerian Heart Foundation, Osogbo, Nigeria

${ }^{10}$ Healthy People Rwanda, Kigali, Rwanda

${ }^{11}$ Icahn School of Medicine, The Mount Sinai Hospital, New York, USA

${ }^{12}$ Department of Preventive Medicine and Medicine-Cardiology, Northwestern University Feinberg School of Medicine, Chicago, USA

${ }^{13}$ Department of Medicine and Population Health Research Institute, McMaster University and Hamilton Health Sciences, Hamilton, Ontario, Canada

${ }^{14}$ Centro de Investigación para la Epidemia del Tabaquismo, Montevideo, Uruguay

Acknowledgements The KOMPLY Project team is sincerely thankful to World Heart Federation Emerging Leaders Program for the financial support to conduct this study. We also thank Professor William Bazeyo (Dean Makerere University School of Public Health and the CTCA Centre Director) and the entire Center for Tobacco Control in Africa (CTCA) for their support on this research project. We also extend our gratitude to Professor Geoffrey Fong (University of Waterloo, Canada), Professor David Guwatudde (Makerere University School of Public Health), Dr Sean Semple (Division of Applied Health Sciences, University of Aberdeen) and Dr Sheila Ndyanabangi (Ministry of Heath Uganda) for their guidance and support throughout the development, data collection and dissemination of this research project. The team acknowledges the 3 sets of fieldworkers who collected the data for this study as well as the ITC Project and the Institute of Global Tobacco Control of the Johns
Hopkins School of Public Health for the use of their Sidepack machines. Finally, the authors thank the Reviewers for their guidance and insight.

Contributors SG, KNN, SNK, GO, LR, KKCH, AEN, ASO, JCR, SK and EB conceived and designed the study. MDH provided critical review of the study design protocol. SG drafted the manuscript and created the tables and figures. SG, KNN, SNK, GO, LR, KKCH, AEN, ASO, JCR, SK, SY and EB read and critically revised the manuscript.

Funding The KOMPLY Smoke-Free Emerging Leaders Project was supported by the World Heart Federation. Additionally, SG was supported by a Career Development Award from the Canadian Cancer Society (\#703858). LR was supported by a Postdoctoral Fellowship from the Department of Preventive and Social Medicine, University of Otago.

Competing interests None declared.

Ethics approval All procedures performed in studies involving human participants were in accordance with the ethical standards of the 1964 Declaration of Helsinki and its later amendments or comparable ethical standards. The study protocol was approved by the Ethics Committee at Makerere University School of Public Health and registered with the Uganda National Council for Science and Technology.

Provenance and peer review Not commissioned; externally peer reviewed.

Data sharing statement The data are owned and shared by the Centre for Tobacco Control in Africa (CTCA) and Makerere University School of Public Health (MakSPH). Requests for data sharing can be made to Kellen Namusisi Nyamurungi, CTCA: nyamurungik@ctc-africa.org or Steven Kabwama Ndugwa, MakSPH: skabwama@musph.ac.ug.

Open Access This is an Open Access article distributed in accordance with the Creative Commons Attribution Non Commercial (CC BY-NC 4.0) license, which permits others to distribute, remix, adapt, build upon this work non-commercially, and license their derivative works on different terms, provided the original work is properly cited and the use is non-commercial. See: http://creativecommons.org/ licenses/by-nc/4.0/

(c) Article author(s) (or their employer(s) unless otherwise stated in the text of the article) 2018. All rights reserved. No commercial use is permitted unless otherwise expressly granted.

\section{REFERENCES}

1. National Cancer Institute. Secondhand smoke and cancer. Bethesda, MD: National Cancer Institute, 2011. https://www.cancer.gov/about- 
cancer/causes-prevention/risk/tobacco/second-hand-smoke-factsheet (accessed 27 Jun 2017).

2. Centers for Disease Control. Health Effects of Secondhand Smoke. GA, USA: Centers for Disease Control, 2017. https://www.cdc.gov/ tobacco/data_statistics/fact_sheets/secondhand_smoke/health_ effects/ (accessed 27 Jun 2017).

3. World Health Organization. Tobacco. Geneva, Switzerland: World Health Organization, 2017. http://www.who.int/mediacentre/ factsheets/fs339/en/ (accessed 27 Jun 2017).

4. International Agency for Research on Cancer (IARC). Evaluating the Effectiveness of Smoke-free Policies. IARC Handbooks of Cancer Prevention, Tobacco Control. Lyon, France: WHO Press, 2009:13.

5. The United States Environmental Protection Agency (US EPA). Revised PM2.5 AQI breakpoints. 2012. http://aqicn.org/faq/2013-0909/revised-pm25-aqi-breakpoints/ (accessed 27 Jun 2017).

6. Hopkins DP, Razi S, Leeks KD, et al. Smokefree policies to reduce tobacco use: a systematic review. Am J Prev Med 2010;38:S275-89.

7. Tan CE, Glantz SA. Association between smoke-free legislation and hospitalizations for cardiac, cerebrovascular, and respiratory diseases: a meta-analysis. Circulation 2012;126:2177-83.

8. Kalkhoran S, Sebrié EM, Sandoya E, et al. Effect of uruguay's national $100 \%$ Smokefree law on emergency visits for bronchospasm. Am J Prev Med 2015;49:85-8.

9. U.S. National Cancer Institute and World Health Organization. The Economics of Tobacco and Tobacco Control. National Cancer Institute Tobacco Control Monograph 21. Bethesda, MD: U.S. Department of Health and Human Services, National Institutes of Health, National Cancer Institute, 2016. https://cancercontrol.cancer. gov/brp/tcrb/monographs/21/docs/m21_complete.pdf. (accessed 27 Jun 2017).

10. Navas-Acien A, Peruga A, Breysse P, et al. Secondhand tobacco smoke in public places in Latin America, 2002-2003. JAMA 2004;291:2741-5.

11. Nebot M, López MJ, Gorini G, et al. Environmental tobacco smoke exposure in public places of European cities. Tob Control 2005;14:60-3.

12. Lopez MJ, Nebot M, Albertini M, et al. Secondhand smoke exposure in hospitality venues in Europe. Environ Health Perspect 2008;116:1469-72.

13. Wakefield M, Trotter L, Cameron M, et al. Association between exposure to workplace secondhand smoke and reported respiratory and sensory symptoms: cross-sectional study. J Occup Environ Med 2003;45:622-7.

14. Travers MJ, Vogl L. Department of Health Behavior and the Aerosol Pollution Exposure Research Laboratory (APERL). Effect of smokefree air law on indoor air quality. NY, USA: Roswell Park Research Institute, 2012

15. Apelberg BJ, Hepp LM, Avila-Tang E, et al. Environmental monitoring of secondhand smoke exposure. Tob Control 2013;22:147-55.

16. Repace J. Air quality in Michigan Restaurants and Casinos. Before and After. MI, USA: The Michigan Department Of Community Health, Tobacco Section, 2011.

17. Yamato $\mathrm{H}$, Mori $\mathrm{N}$, Horie R, et al. Designated smoking areas in streets where outdoor smoking is banned. Kobe $\mathrm{J} \mathrm{Med} \mathrm{Sci}$ 2013;59:E93-105.

18. Ward M, Currie LM, Kabir Z, et al. The efficacy of different models of smoke-free laws in reducing exposure to second-hand smoke: a multi-country comparison. Health Policy 2013;110:207-13.

19. Agbenyikey W, Wellington E, Gyapong J, et al. Secondhand tobacco smoke exposure in selected public places (PM2.5 and air nicotine) and non-smoking employees (hair nicotine) in Ghana. Tob Control 2011;20:107-11.

20. Fu M, Martínez-Sánchez JM, Galán I, et al. Variability in the correlation between nicotine and PM2.5 as airborne markers of second-hand smoke exposure. Environ Res 2013;127:49-55.

21. Hyland A, Travers MJ, Dresler C, et al. A 32-country comparison of tobacco smoke derived particle levels in indoor public places. Tob Control 2008;17:159-65

22. Emerging Leaders KOMPLY Smoke-free Project. Results from an evaluation of compliance, knowledge, and attitudes related to the $100 \%$ smoke-free law in bars and restaurants in Kampala, Uganda. Kampala, Uganda: Centre for Tobacco Control in Africa, 2017. http://ctc-africa.org/images/docments/reports/KOMPLY_report.pdf (accessed 27 Jun 2017).

23. World Health Organization. WHO report on the global tobacco epidemic, 2015: Raising taxes on tobacco. Geneva, Switzerland: WHO Press, 2015. http://www.who.int/tobacco/global_report/2015/ report/en/ (accessed 27 Jun 2017).

24. Kaai SC, Fong GT, Burhoo P, et al. Prevalence of smoking in public venues and support for smoke-free regulations among smokers and non-smokers: Findings from the ITC African countries. 2017;POS2-
74:172. http://c.ymcdn.com/sites/www.srnt.org/resource/resmgr/ Conferences/2016_Annual_Meeting/Program/FINAL_SRNT Abstract_WEB02171.pdf (accessed 27 Jun 2017).

25. Ministry of Health Republic of Uganda. The Tobacco Control Act. Kampala Uganda: Ministry of Health Republic of Uganda, 2015. http://health.go.ug/content/tobacco-control-act-2015 (accessed 27 Jun 2017).

26. Ministry of Health Uganda, Uganda Bureau of Statistics, World Health. Uganda Global Adult Tobacco Survey: Country Report, 2013. http://www.who.int/tobacco/surveillance/survey/gats/uga/en/ (accessed 27 Jun 2017).

27. Country Meters. Uganda Population, 2017. http://countrymeters.info/ en/Uganda (accessed 27 Jun 2017).

28. https://www.google.ca/maps/place/Uganda/@1.3671056,30. 0586288,7z/data=!3m1!4b1!4m5!3m4!1s0x1771a69f6499f945: 0x874155ce43014549!8m2!3d1.373333!4d32.290275 (accessed 13 Nov 2017).

29. Bennett S, Woods T, Liyanage WM, et al. A simplified general method for cluster-sample surveys of health in developing countries. World Health Stat Q 1991;44:98-106.

30. World Health Organization. Immunization Cluster survey Reference Manual. Geneva, Switzerland: Department of Immunization,Vaccines and Biologicals, World Health Organization, 2005. http://apps. who. int/iris/handle/10665/69087 (accessed 27 Jun 2017).

31. Kumar R, Goel S, Harries AD, et al. How good is compliance with smoke-free legislation in India? Results of 38 subnational surveys. Int Health 2014;6:189-95.

32. Uganda Bureau of Statistic. National Population and Housing Census-Main Report. Kampala, Uganda, 2014. http://www.ubos.org/ onlinefiles/uploads/ubos/NPHC/2014\%20National\%20Census\% 20Main\%20Report.pdf (accessed 27 Jun 2017).

33. U.S. Environmental Protection Agency. Particulate matter (PM2.5) speciation guidance. Research Triangle Park, NC: Monitoring and Quality Assurance Group, Emissions, Monitoring, and Analysis Division, Office of Air Quality Planning and Standards, 1999.

34. International Union Against Tuberculosis and Lung Disease, Campaign for Tobacco-free Kids, John Hopkins Bloomberg Schoo of Public Health. A "How-to" Guide for Conducting Compliance Studies. Assessing Compliance with Smoke-Free Laws. 2nd edn, 2014. http://global.tobaccofreekids.org/files/pdfs/en/smoke_free_ compliance guide.pdf (accessed 27 Jun 2017).

35. Travers MJ, Vogl L. Indoor air monitoring protocol. Buffalo, New York: Roswell Park Cancer Institute, 2006. http://tobaccofreeair.org/ documents/US_Air_Monitoring_Protocol_Jun2006.pdf (accessed 2 May 2017).

36. Vardavas $\mathrm{Cl}$, Kondilis B, Travers MJ, et al. Environmental tobacco smoke in hospitality venues in Greece. BMC Public Health 2007;7:302

37. Travers MJ, Nayak NS, Annigeri VB, et al. Indoor air quality due to secondhand smoke: Signals from selected hospitality locations in rural and urban areas of Bangalore and Dharwad districts in Karnataka, India. Indian J Cancer 2015;52:708-13.

38. Licht AS, Hyland A, Travers MJ, et al. Secondhand smoke exposure levels in outdoor hospitality venues: a qualitative and quantitative review of the research literature. Tob Control 2013;22:172-9.

39. Jackson-Morris A, Bleymann K, Lyall E, et al. Low-cost air quality monitoring methods to assess compliance with Smoke-Free regulations: a multi-center study in six low- and middle-income countries. Nicotine Tob Res 2016;18:1258-64.

40. TSI Inc. SidePak ${ }^{T M}$ Personal Aerosol Monitor AM510. http://www.tsi. com/sidepak-personal-aerosol-monitor-am510/ (accessed 27 Jun 2017).

41. US Department of Health and Human Services. The health consequences of involuntary exposure to tobacco smoke: a report of the Surgeon General. Atlanta, GA: US Department of Health and Human Services, Centers for Disease Control and Prevention, Coordinating Centerfor Health Promotion, National Center for Chronic Disease Prevention and Health Promotion,Office on Smoking and Health, 2006:709.

42. US Department of Health and Human Services. The health consequences of smoking - 50 years of progress. A report of the Surgeon General. Washington DC: U.S. Department of Health \& Human Services, 2014. https://www. surgeongeneral.gov/library/reports/50-years-of-progress/ (accessed 27 Jun 2017).

43. World Health Organization. Guidelines for implementation of Article 8 of the WHO FCTC (Protection from exposure to tobacco smoke). http://www.who.int/fctc/cop/art\%208\%20guidelines_english.pdf? ua=1 (accessed 27 Jun 2017)

44. Ay P, Evrengil E, Guner M, et al. Noncompliance to smoke-free law: which hospitality premises are more prone? Public Health 2016;141:1-6. 
45. Reis MF, Namorado S, Aguiar P, et al. Patterns of adherence to and compliance with the Portuguese smoke-free law in the leisurehospitality sector. PLoS One 2014;9:e102421.

46. Barnoya J, Monzon JC, Briz P, et al. Compliance to the smoke-free law in Guatemala 5-years after implementation. BMC Public Health 2016;16:318.

47. Buettner-Schmidt K, Lobo ML, Travers MJ, et al. Tobacco smoke exposure and impact of smoking legislation on rural and non-rural hospitality venues in North Dakota. Res Nurs Health 2015;38:268-77.

48. Brown A, Moodie C, Hastings G. A longitudinal study of policy effect (smoke-free legislation) on smoking norms: ITC Scotland/United Kingdom. Nicotine Tob Res 2009;11:924-32.

49. Centers for Disease Control and Prevention. Smoking \& Tobacco Use. Smoke-Free Policies Receive Public Support. 2016. https:// www.cdc.gov/tobacco/data statistics/fact sheets/secondhand smoke/protection/public_support/ (accessed 27 Jun 2017).

50. Fong GT, Hyland A, Borland R, et al. Reductions in tobacco smoke pollution and increases in support for smoke-free public places following the implementation of comprehensive smoke-free workplace legislation in the Republic of Ireland: findings from the ITC Ireland/UK Survey. Tob Control 2006;15(Suppl 3):iii51-8.

51. Fong GT, Craig LV, Guignard R, et al. Evaluating the effectiveness of France's indoor Smoke-Free Law 1 year and 5 years after implementation: findings from the ITC France survey. PLoS One 2013;8:e66692.
52. Cooper J, Borland $\mathrm{R}$, Yong $\mathrm{HH}$, et al. Compliance and support for bans on smoking in licensed venues in Australia: findings from the International Tobacco Control Four-Country Survey. Aust N Z J Public Health 2010;34:379-85.

53. Hyland A, Hassan LM, Higbee C, et al. The impact of smokefree legislation in Scotland: results from the Scottish ITC: Scotland/ UK longitudinal surveys. Eur J Public Health 2009;19:198-205.

54. Li Q, Hyland A, O'Connor R, et al. Support for smoke-free policies among smokers and non-smokers in six cities in China: ITC China Survey. Tob Control 2010;19(Suppl 2):i40-6.

55. European Commission. Tobacco in the EU: Exposure to second hand smoke reduced, but still too high, says Commission report. http:// europa.eu/rapid/press-release_IP-13-147_en.htm (accessed 27 Jun 2017).

56. Aherrera A, Çarkoğlu A, Hayran M, et al. Factors that influence attitude and enforcement of the smoke-free law in Turkey: a survey of hospitality venue owners and employees. Tob Control 2017;26:540-7.

57. The International Tobacco Control Policy Evaluation Project. Smokefree Policies. ITC Cross-Country Comparison Report, 2012. http:// www.itcproject.org/files/ITC_CrossCountry-Smoke-free_Final-web. pdf (accessed 07 Sept 2017).

58. Scollo M, Lal A, Hyland A, et al. Review of the quality of studies on the economic effects of smoke-free policies on the hospitality industry. Tob Control 2003;12:13-20. 